Infinite Dimensional Analysis, Quantum Probability and Related Topics

Vol. 9, No. 1 (2006) 129-147

(C) World Scientific Publishing Company

\title{
RENORMALIZED POWERS OF QUANTUM WHITE NOISE
}

\author{
LUIGI ACCARDI \\ Centro Vito Volterra, Università di Roma Tor Vergata, \\ Via Columbia, 2, 00133 Roma, Italy \\ accardi@volterra.uniroma2.it \\ ANDREAS BOUKAS \\ Department of Mathematics and Natural Sciences, \\ American College of Greece, Aghia Paraskevi, \\ 15342 Athens, Greece \\ andreasboukas@acgmail.gr \\ UWE FRANZ \\ Département de Mathématiques de Besançon, \\ Université de Franche-Comte, 16, route de Gray, \\ 25030 Besançon Cedex, France \\ uwe.franz@math.univ-fcomte.fr \\ Received 10 January 2005 \\ Communicated by Y. G. Lu
}

We prove some no-go theorems on the existence of a Fock representation of the *-Lie algebra generated by $B_{k}^{n}(t)=\int_{0}^{t} b_{s}^{\dagger^{n}} b_{s}^{k} d s$, where $b_{s}^{\dagger}, b_{s}$ are the Hida white noise densities. In particular we prove the nonexistence of such a representation for any $*$-Lie algebra containing $b_{s}^{3}$. This drastic difference with the quadratic case proves the necessity of investigating different renormalization rules for the case of higher powers of white noise.

Keywords: Quantum white noise; Fock representation.

AMS Subject Classification: 17B65, 60H40, 81S25

\section{Introduction}

The standard boson white noise Lie algebra is defined by its generators, $b_{t}, b_{s}^{\dagger}, 1$ (central element) in the sense of operator valued distributions over $\mathbb{R}^{d}$ on an appropriate space of test functions (cf. Ref. 5 for this notion), and by the commutation relations:

$$
\begin{aligned}
& {\left[b_{t}, b_{s}^{\dagger}\right]=\delta(t-s) 1 ; \quad s, t \geq 0} \\
& {\left[b_{t}^{\dagger}, b_{s}^{\dagger}\right]=\left[b_{t}, b_{s}\right]=0 .}
\end{aligned}
$$


Our goal in this paper is to investigate the possibility of giving a meaning to the higher powers of white noise, i.e. the symbolic expressions

$$
b_{t}^{n}, b_{s}^{\dagger^{k}} ; \quad n, k \in\{0,1,2, \ldots\}
$$

as generators of a Lie algebra with commutation relations obtained by formally extending, using the Leibnitz rule, the relations (1.1), (1.2) to the symbols (1.3). As shown in the following section, this formal extension involves higher powers of the $\delta$ function and some renormalization is required to give a meaning to these powers. As shown in Ref. 9 the renormalization procedure usually adopted in physics (introduce a cutoff and then take limits to remove it) can only lead to trivial (i.e. Gaussian) limits.

A new approach to the problem was proposed in Ref. 5 where it was suggested to renormalize the commutation relations of the higher powers of white noise and then to look for a Fock representation (cf. Definition 4.1 below) of the ${ }^{*}$-Lie algebra obtained in this way.

Since the Lie algebra commutation relations, together with the Fock prescription, uniquely determine the statistics, i.e. the scalar product in the representation space, the problem is reduced to the proof that the combination of these two ingredients leads to a positive-definite sesquilinear form.

This leads to the problem of finding a definition of these renormalized powers of quantum white noise (RPQWN) with the additional properties that:

(i) The resulting commutation relations define a *-Lie algebra structure which, when the test function space is restricted to the linear span of the characteristic functions of $n$ mutually disjoint open sets $(n \in \mathbb{N})$, becomes isomorphic to the full oscillator algebra, i.e. the *-Lie algebra generated by the powers $a_{\alpha}^{h}, a_{\beta}^{+k}$ of the generators of the Schrödinger representation of the $n$-dimesional Heisenberg-Weyl Lie algebra with central element $E$ :

$$
\left[a_{\alpha}, a_{\beta}^{+}\right]=c^{2} \delta_{\alpha, \beta} E ; \quad \alpha, \beta=1, \ldots, n ; \quad c \in \mathbb{R} \backslash\{0\} .
$$

This condition is needed to guarantee that the distribution of $\left(b_{t}+b_{s}^{\dagger}\right)^{k}$ in the Fock representation (if it exists) will effectively be the $k$ th power of a Gaussian distribution.

(ii) This *-Lie algebra admits a Fock representation.

(iii) The space of test functions over which the RPQWN are defined contains characteristic functions of open sets of arbitrarily small (Lebesgue) volume.

This program was successfully realized in Ref. 5 for the second-order powers and lead to an unexpected connection with the Meixner distributions in Ref. 3 (cf. Ref. 1 for more information on this connection). In this paper we begin to study the problem for the higher powers of white noise. In order to state more precisely the problem, let us recall that the main result of Ref. 3 can be formulated as follows: the current algebra over the Lie algebra $\operatorname{sl}(2, \mathbb{R})$ admits a Fock representation (hence, up to isomorphism, only one). 
Now $\operatorname{sl}(2, \mathbb{R})$ is isomorphic to the Lie algebra generated by $a^{2}, a^{+2}, N:=a^{+} a$ and the Heisenberg-Weyl algebra is generated by $a, a^{+}, 1$ (the symbols are referred to the Schrödinger representation. Since the current algebra over the HeisenbergWeyl algebra (given by (1.1) and (1.2)) has the usual Fock representation, it is natural to ask oneself whether the current algebra over the combined Lie algebra, i.e. the one generated by

$$
a, a^{+}, 1, a^{2}, a^{+2}, N:=a^{+} a
$$

(and called the Schrödinger algebra) has a Fock representation.

Sniady first raised this problem and proved in Ref. 11 that, surprisingly, the answer is negative. Accardi, Franz and Skeide extended this result in Ref. 3 by showing that there can exist no Lévy process on the Schrödinger algebra whose restriction to the Heisenberg-Weyl algebra is not identically zero and whose restriction to $\operatorname{sl}(2, \mathbb{R})$ gives the Fock representation of the current algebra over $\operatorname{sl}(2, \mathbb{R})$.

From these results one deduces that: there exist subalgebras of the full oscillator algebra such that the corresponding current algebras separately admit a Fock representation, but not jointly, i.e. the current algebra over the Lie algebra generated by their union does not admit a Fock representation. In particular the current algebra over the full oscillator algebra does not admit a Fock representation.

On the other hand, since the *-Lie algebra generated by the powers $a_{\alpha}^{h}, a_{\beta}^{+k}$ with $h, k \geq 3$ has zero intersection with the Schrödinger algebra, one could hope that at least the current algebra over this Lie algebra exists.

In this paper we prove that this is not the case. More precisely we prove two no-go theorems for the full oscillator algebra which imply that the current algebra over the Lie algebra generated by $a^{3}, a^{+3}$ does not admit a Fock representation.

The last section of this paper is devoted to a new no-go theorem for the Schrödinger algebra which excludes the existence not only of the Fock representation, but also of a large class of Gaussian non-Fock representations, including the finite temperature representations constructed in Ref. 7.

\section{Formal White Noise Commutators}

Lemma 2.1. For $l \in \mathbb{N}$ denote $\delta^{l}(t-s)$ the formal lth power of the $\delta$-function $\left(\delta^{0}:=1\right)$. For all $t, s \in \mathbb{R}_{+}$and $n, k \geq 0$, the rules (1.1), (1.2) imply that

$$
\left[b_{t}^{n}, b_{s}^{\dagger^{k}}\right]=\epsilon_{n, 0} \epsilon_{k, 0} \sum_{l \geq 1}\left(\begin{array}{c}
n \\
l
\end{array}\right) k^{(l)} b_{s}^{\dagger^{k-l}} b_{t}^{n-l} \delta^{l}(t-s)
$$

where

$$
\begin{array}{ll}
\left(\begin{array}{c}
n \\
l
\end{array}\right)=\frac{n !}{(n-l) ! l !}, & l \in\{0,1, \ldots, n\} \\
\left(\begin{array}{c}
n \\
l
\end{array}\right)=0, & n<l .
\end{array}
$$


Moreover, for $k=0,1,2, \ldots$,

$$
k^{(l)}= \begin{cases}k(k-1)(k-2) \cdots(k-l+1) & \text { if } l-1<k, \\ 0 & \text { if } l-1 \geq k .\end{cases}
$$

In particular

$$
k^{(0)}=1
$$

Proof. We will let $k$ be arbitrary and use induction on $n$. The cases $n=0$ and/or $k=0$ are obvious. For $n=1$ and $k>0$ we have

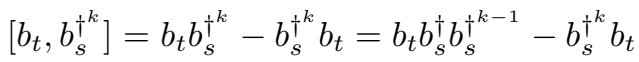

$$
\begin{aligned}
& =\left(b_{s}^{\dagger} b_{t}+\delta(t-s)\right) b_{s}^{\dagger^{k-1}}-b_{s}^{\dagger^{k}} b_{t} \\
& =b_{s}^{\dagger} b_{t} b_{s}^{\dagger^{k-1}}+\delta(t-s) b_{s}^{\dagger^{k-1}}-b_{s}^{\dagger^{k}} b_{t} \\
& =b_{s}^{\dagger} b_{t} b_{s}^{\dagger} b_{s}^{\dagger^{k-2}}+\delta(t-s) b_{s}^{\dagger^{k-1}}-b_{s}^{\dagger^{k}} b_{t} \\
& =b_{s}^{\dagger}\left(b_{s}^{\dagger} b_{t}+\delta(t-s)\right) b_{s}^{\dagger^{k-2}}+\delta(t-s) b_{s}^{\dagger^{k-1}}-b_{s}^{\dagger^{k}} b_{t} \\
& =b_{s}^{\dagger^{2}} b_{t} b_{s}^{\dagger^{k-2}}+\delta(t-s) b_{s}^{\dagger^{k-1}}+\delta(t-s) b_{s}^{\dagger^{k-1}}-b_{s}^{\dagger^{k}} b_{t} \\
& =b_{s}^{\dagger^{2}} b_{t} b_{s}^{\dagger^{k-2}}+2 \delta(t-s) b_{s}^{\dagger^{k-1}}-b_{s}^{\dagger^{k}} b_{t} \\
& =b_{s}^{\dagger^{2}} b_{t} b_{s}^{\dagger} b_{s}^{\dagger^{k-3}}+2 \delta(t-s) b_{s}^{\dagger^{k-1}}-b_{s}^{\dagger^{k}} b_{t} \\
& =b_{s}^{\dagger^{2}}\left(b_{s}^{\dagger} b_{t}+\delta(t-s)\right) b_{s}^{\dagger^{k-3}}+2 \delta(t-s) b_{s}^{\dagger^{k-1}}-b_{s}^{\dagger^{k}} b_{t} \\
& =b_{s}^{\dagger^{3}} b_{t} b_{s}^{\dagger^{k-3}}+3 \delta(t-s) b_{s}^{\dagger^{k-1}}-b_{s}^{\dagger^{k}} b_{t} \\
& =b_{s}^{\dagger^{k}} b_{t}+k \delta(t-s) b_{s}^{\dagger^{k-1}}-b_{s}^{\dagger^{k}} b_{t} \\
& =k \delta(t-s) b_{s}^{\dagger^{k-1}} \\
& =\sum_{l \geq 1}\left(\begin{array}{l}
1 \\
l
\end{array}\right) k^{(l)} b_{s}^{\dagger^{k-l}} b_{t}^{1-l} \delta^{l}(t-s) \text {. }
\end{aligned}
$$

Thus (2.1) is true for $n=1$. Suppose that it is true for $n=m$. We will show that it is true for $n=m+1$. We have

$$
\begin{aligned}
b_{t}^{m+1} b_{s}^{\dagger^{k}} & =b_{t} b_{t}^{m} b_{s}^{\dagger^{k}} \\
& =b_{t}\left(b_{s}^{\dagger^{k}} b_{t}^{m}+\sum_{l \geq 1}\left(\begin{array}{c}
m \\
l
\end{array}\right) k^{(l)} b_{s}^{\dagger^{k-l}} b_{t}^{m-l} \delta^{l}(t-s)\right) \\
& =b_{s}^{\dagger^{k}} b_{t}^{m+1}+k b_{s}^{\dagger^{k-1}} b_{t}^{m} \delta(t-s)+\sum_{l \geq 1}\left(\begin{array}{c}
m \\
l
\end{array}\right) k^{(l)} b_{t} b_{s}^{\dagger^{k-l}} b_{t}^{m-l} \delta^{l}(t-s)
\end{aligned}
$$




$$
\begin{aligned}
= & b_{s}^{\dagger^{k}} b_{t}^{m+1}+k b_{s}^{\dagger^{k-1}} b_{t}^{m} \delta(t-s)+\sum_{l \geq 1}\left(\begin{array}{c}
m \\
l
\end{array}\right) k^{(l)}\left(b_{s}^{\dagger^{k-l}} b_{t}\right. \\
& \left.+(k-l) b_{s}^{\dagger^{k-l-1}} \delta(t-s)\right) b_{t}^{m-l} \delta^{l}(t-s) \\
= & b_{s}^{\dagger^{k}} b_{t}^{m+1}+k b_{s}^{\dagger^{k-1}} b_{t}^{m} \delta(t-s)+\sum_{l \geq 1}\left(\begin{array}{c}
m \\
l
\end{array}\right) k^{(l)} b_{s}^{\dagger^{k-l}} b_{t}^{m-l+1} \delta^{l}(t-s) \\
& +\sum_{l \geq 1}\left(\begin{array}{c}
m \\
l
\end{array}\right) k^{(l)}(k-l) b_{s}^{\dagger^{k-l-1}} b_{t}^{m-l} \delta^{l+1}(t-s)
\end{aligned}
$$

which, upon letting $L=l+1$ in the last sum, becomes

$$
\begin{aligned}
= & b_{s}^{\dagger^{k}} b_{t}^{m+1}+k b_{s}^{\dagger^{k-1}} b_{t}^{m} \delta(t-s)+\sum_{l \geq 1}\left(\begin{array}{c}
m \\
l
\end{array}\right) k^{(l)} b_{s}^{\dagger^{k-l}} b_{t}^{m-l+1} \delta^{l}(t-s) \\
& +\sum_{L \geq 2}\left(\begin{array}{c}
m \\
L-1
\end{array}\right) k^{(L-1)}(k-L+1) b_{s}^{\dagger^{k-L}} b_{t}^{m-L+1} \delta^{L}(t-s) \\
= & b_{s}^{\dagger^{k}} b_{t}^{m+1}+k b_{s}^{\dagger^{k-1}} b_{t}^{m} \delta(t-s)+m k b_{s}^{\dagger^{k-1}} b_{t}^{m} \delta(t-s) \\
& +\sum_{l=2}^{m}\left(\begin{array}{c}
m \\
l
\end{array}\right)+\left(\begin{array}{c}
m \\
l-1
\end{array}\right) k^{(l)} b_{s}^{\dagger^{k-l}} b_{t}^{m-l+1} \delta^{l}(t-s) \\
& +k^{(m)}(k-m) b_{s}^{\dagger^{k-m-1}} \delta^{m}(t-s) \\
= & b_{s}^{\dagger^{k}} b_{t}^{m+1}+(m+k) b_{s}^{\dagger^{k-1}} b_{t}^{m} \delta(t-s) \\
& +\sum_{l=2}^{m}\left(\left(\begin{array}{c}
m \\
l
\end{array}\right)+\left(\begin{array}{c}
m \\
l-1
\end{array}\right)\right) k^{(l)} b_{s}^{\dagger^{k-l}} b_{t}^{m-l+1} \delta^{l}(t-s) \\
& +k^{(m+1)} b_{s}^{\dagger^{k-m-1}} \delta^{m}(t-s) .
\end{aligned}
$$

Using $\left(\begin{array}{c}m \\ l\end{array}\right)+\left(\begin{array}{c}m \\ l-1\end{array}\right)=\left(\begin{array}{c}m+1 \\ l\end{array}\right)$ this becomes

$$
\begin{aligned}
= & b_{s}^{\dagger^{k}} b_{t}^{m+1}+(m+1) k b_{s}^{\dagger^{k-1}} b_{t}^{m} \delta(t-s) \\
& +\sum_{l=2}^{m}\left(\begin{array}{c}
m+1 \\
l
\end{array}\right) k^{(l)} b_{s}^{\dagger^{k-l}} b_{t}^{m-l+1} \delta^{l}(t-s) \\
& +k^{(m+1)} b_{s}^{\dagger^{k-m-1}} \delta^{m}(t-s) \\
= & b_{s}^{\dagger^{k}} b_{t}^{m+1}+\sum_{l \geq 1}\left(\begin{array}{c}
m+1 \\
l
\end{array}\right) k^{(l)} b_{s}^{\dagger^{k-l}} b_{t}^{m-l+1} \delta^{l}(t-s) .
\end{aligned}
$$


Lemma 2.2. For all $t, s \in \mathbb{R}_{+}$and $n, k, N, K \geq 0$,

$b_{t}^{\dagger^{n}} b_{t}^{k} b_{s}^{\dagger^{N}} b_{s}^{K}=b_{t}^{\dagger^{n}} b_{s}^{\dagger^{N}} b_{t}^{k} b_{s}^{K}+\epsilon_{k, 0} \epsilon_{N, 0} \sum_{l \geq 1}\left(\begin{array}{c}k \\ l\end{array}\right) N^{(l)} b_{t}^{\dagger^{n}} b_{s}^{\dagger^{N-l}} b_{t}^{k-l} b_{s}^{K} \delta^{l}(t-s)$.

\section{Proof.}

$$
\begin{aligned}
b_{t}^{\dagger^{n}} b_{t}^{k} b_{s}^{\dagger^{N}} b_{s}^{K} & =b_{t}^{\dagger^{n}}\left(b_{t}^{k} b_{s}^{\dagger^{N}}\right) b_{s}^{K} \\
& =b_{t}^{\dagger^{n}}\left(\left[b_{t}^{k}, b_{s}^{\dagger^{N}}\right]+b_{s}^{\dagger^{N}} b_{t}^{k}\right) b_{s}^{K} \\
& =b_{t}^{\dagger^{n}}\left(\epsilon_{k, 0} \epsilon_{N, 0} \sum_{l \geq 1}\left(\begin{array}{c}
k \\
l
\end{array}\right) N^{(l)} b_{s}^{\dagger^{N-l}} b_{t}^{k-l} \delta^{l}(t-s)+b_{s}^{\dagger^{N}} b_{t}^{k}\right) b_{s}^{K} \\
& =\epsilon_{k, 0} \epsilon_{N, 0} \sum_{l \geq 1}\left(\begin{array}{c}
k \\
l
\end{array}\right) N^{(l)} b_{t}^{n} b_{s}^{\dagger^{N-l}} b_{t}^{k-l} b_{s}^{K} \delta^{l}(t-s)+b_{t}^{n} b_{s}^{\dagger^{N}} b_{t}^{k} b_{s}^{K} \\
& =\epsilon_{k, 0} \epsilon_{N, 0} \sum_{l \geq 1}\left(\begin{array}{c}
k \\
l
\end{array}\right) N^{(l)} b_{t}^{n} b_{s}^{\dagger^{N-l}} b_{t}^{k-l} b_{s}^{K} \delta^{l}(t-s)+b_{t}^{\dagger^{n}} b_{s}^{\dagger^{N}} b_{t}^{k} b_{s}^{K} .
\end{aligned}
$$

Lemma 2.3. For all $t, s \in \mathbb{R}_{+}$and $n, k, N, K \geq 0$

$$
\begin{aligned}
{\left[b_{t}^{\dagger^{n}} b_{t}^{k}, b_{s}^{\dagger^{N}} b_{s}^{K}\right]=} & \epsilon_{k, 0} \epsilon_{N, 0} \sum_{l \geq 1}\left(\begin{array}{c}
k \\
l
\end{array}\right) N^{(l)} b_{t}^{\dagger^{n}} b_{s}^{\dagger^{N-l}} b_{t}^{k-l} b_{s}^{K} \delta^{l}(t-s) \\
& -\epsilon_{K, 0} \epsilon_{n, 0} \sum_{L \geq 1}\left(\begin{array}{c}
K \\
L
\end{array}\right) n^{(L)} b_{s}^{\dagger^{N}} b_{t}^{\dagger^{n-L}} b_{s}^{K-L} b_{t}^{k} \delta^{L}(t-s)
\end{aligned}
$$

Proof. The first term on the right-hand side of (2.1) is

$$
\begin{aligned}
& b_{t}^{\dagger^{n}} b_{s}^{\dagger^{N}} b_{t}^{k} b_{s}^{K}=b_{s}^{\dagger^{N}} b_{t}^{\dagger^{n}} b_{s}^{K} b_{t}^{k} \\
& =b_{s}^{\dagger^{N}}\left(\left[b_{t}^{\dagger^{n}}, b_{s}^{K}\right]+b_{s}^{K} b_{t}^{\dagger^{n}}\right) b_{t}^{k} \\
& =b_{s}^{\dagger^{N}}\left(-\left[b_{s}^{K}, b_{t}^{\dagger^{n}}\right]+b_{s}^{K} b_{t}^{\dagger^{n}}\right) b_{t}^{k} \\
& =b_{s}^{\dagger^{N}}\left(-\epsilon_{n, 0} \epsilon_{K, 0} \sum_{l \geq 1}\left(\begin{array}{c}
K \\
l
\end{array}\right) n^{(l)} b_{t}^{\dagger^{n-l}} b_{s}^{K-l} \delta^{l}(t-s)+b_{s}^{K} b_{t}^{\dagger^{n}}\right) b_{t}^{k} \\
& =-\epsilon_{n, 0} \epsilon_{K, 0} \sum_{l \geq 1}\left(\begin{array}{c}
K \\
l
\end{array}\right) n^{(l)} b_{s}^{\dagger^{N}} b_{t}^{\dagger^{n-l}} b_{s}^{K-l} b_{t}^{k} \delta^{l}(t-s)+b_{s}^{\dagger^{N}} b_{s}^{K} b_{t}^{\dagger^{n}} b_{t}^{k}
\end{aligned}
$$

from which (2.3) follows by substituting into (2.2). 


\section{Renormalized White Noise Commutators}

Motivated by Ref. 5 we introduce the renormalization

$$
\delta^{l}(t-s)=c_{l-1} \delta(t-s), \quad l \geq 1, \quad c_{0}=1,
$$

where the $c_{l-1}$ are constants to be defined and

$$
c_{l-1}>0, \quad \forall l .
$$

Let $f$ be a test function. We define the symbols

$$
B_{k}^{n}(f)=\int_{\mathbb{R}} f(s) b_{s}^{\dagger^{n}} b_{s}^{k} d s
$$

with involution

$$
\left(B_{k}^{n}(f)\right)^{*}=B_{n}^{k}(\bar{f})
$$

and with

$$
B_{0}^{0}(f)=\int_{\mathbb{R}} f(s) d s 1=: \mu(f),
$$

where 1 is the identity operator. Multiplying both sides of (2.3) by test functions $f(t) \bar{g}(s)$ and formally integrating the resulting identity (i.e. taking $\iint \cdots d s d t$ ), we obtain the commutation relations

$$
\left[B_{K}^{N}(\bar{g}), B_{k}^{n}(f)\right]=\sum_{L \geq 1} b_{L}(K, n) B_{K+k-L}^{N+n-L}(\bar{g} f)-\sum_{l \geq 1} b_{l}(k, N) B_{K+k-l}^{N+n-l}(\bar{g} f),
$$

where $n, k, N, K \in\{0,1,2, \ldots\}$ and

$$
\epsilon_{n, k}:=1-\delta_{n, k},
$$

where $\delta_{n, k}$ is Kronecker's delta, and

$$
b_{x}(y, z):=\epsilon_{y, 0} \epsilon_{z, 0}\left(\begin{array}{c}
y \\
x
\end{array}\right) z^{(x)} c_{x-1} .
$$

The positive constants $c_{x-1}$ are those in (3.2) and the factorial powers $x^{(y)}$ are defined by:

$$
x^{(y)}=x(x-1) \cdots(x-y+1),
$$

with $x^{(0)}=1$. In particular (3.6) contains the commutation relations of linear white noise:

$$
\begin{aligned}
& {\left[B_{1}^{0}(\bar{g}), B_{0}^{1}(f)\right]=\langle g, f\rangle,} \\
& {\left[B_{1}^{0}(\bar{g}), B_{1}^{1}(f)\right]=B_{1}^{0}(\bar{g} f),} \\
& {\left[B_{1}^{1}(\bar{g}), B_{0}^{1}(f)\right]=B_{0}^{1}(\bar{g} f)}
\end{aligned}
$$


and of the renormalized square of white noise:

$$
\begin{aligned}
& {\left[B_{2}^{0}(\bar{g}), B_{0}^{2}(f)\right]=4 B_{1}^{1}(\bar{g} f)+2 c\langle g, f\rangle,} \\
& {\left[B_{1}^{1}(\bar{g}), B_{2}^{0}(f)\right]=-2 B_{2}^{0}(\bar{g} f),} \\
& {\left[B_{1}^{1}(\bar{g}), B_{0}^{2}(f)\right]=2 B_{0}^{2}(\bar{g} f) .}
\end{aligned}
$$

Choosing in $(3.2)$

$$
c_{l-1}:=c^{l-1}, \quad c>0, \quad \forall l
$$

and fixing all test functions to be multiples of a fixed $f=\chi_{I}$ for some interval $I$, one obtains a Lie algebra isomorphic to the (infinite dimesnional) Lie algebra whose generators are the powers of the generators $a, a^{\dagger}$, of the one-dimensional Schrödinger representation of the CCR. From this, one can deduce that there exist choices of the constant $c$ such that, if one chooses the test function space sufficiently small (e.g. the linear combinations of the characteristic functions of a finite set of mutually disjoint intervals), then the commutation relations (3.6) effectively define a Lie algebra. In what follows we will use the notation

$$
B_{k}^{n}:=B_{k}^{n}\left(\chi_{I}\right),
$$

where $I \subset \mathbb{R}$ with $\mu(I)<+\infty$ is fixed. Moreover, to simplify the notations, we will use the same symbol for the generators of the RPQWN Lie algebra and for their images in a given representation.

Proposition 3.1. For any representation of the $R P Q W N$ Lie algebra, for all $\alpha$, $\beta, \gamma \geq 0$ and all $n \geq 1$, the following identity holds:

$$
\begin{aligned}
& B_{\beta}^{\alpha}\left(B_{0}^{\gamma}\right)^{n}=\left(B_{0}^{\gamma}\right)^{n} B_{\beta}^{\alpha}+\sum_{i=1}^{n}\left(\begin{array}{c}
n \\
i
\end{array}\right) \sum_{L_{1}, L_{2}, \ldots, L_{i} \geq 1} \prod_{j=1}^{i} b_{L_{j}}\left(\beta-\sum_{w=0}^{j-1} L_{w}, \gamma\right) \\
& \times\left(B_{0}^{\gamma}\right)^{n-i} B_{\beta-\sum_{w=0}^{i} L_{w}}^{\alpha+i \gamma-\sum_{w=0}^{i} L_{w}},
\end{aligned}
$$

where $L_{0}=0$.

Proof. For $n=1$ the formula becomes

$$
B_{\beta}^{\alpha} B_{0}^{\gamma}=B_{0}^{\gamma} B_{\beta}^{\alpha}+\sum_{L_{1} \geq 1} b_{L_{1}}(\beta, \gamma) B_{\beta-L_{1}}^{\alpha+\gamma-L_{1}}
$$

which is true by (1.5). Assuming that (3.10) is true for $n=k$ we have

$$
\begin{aligned}
B_{\beta}^{\alpha}\left(B_{0}^{\gamma}\right)^{k+1}= & B_{\beta}^{\alpha}\left(B_{0}^{\gamma}\right)^{k} B_{0}^{\gamma}=\left(\left(B_{0}^{\gamma}\right)^{k} B_{\beta}^{\alpha}+\sum_{i=1}^{k}\left(\begin{array}{c}
k \\
i
\end{array}\right)\right. \\
& \times \sum_{L_{1}, L_{2}, \ldots, L_{i} \geq 1} \prod_{j=1}^{i} b_{L_{j}}\left(\beta-\sum_{w=0}^{j-1} L_{w}, \gamma\right)\left(B_{0}^{\gamma}\right)^{k-i} B_{\left.\beta-\sum_{w=0}^{\alpha+i \gamma-\sum_{w=0}^{i} L_{w}}\right)}^{\alpha} B_{0}^{\gamma}
\end{aligned}
$$


which, by (3.11) and the fact that by (3.6)

$$
\begin{aligned}
& B_{\beta-\sum_{w=0}^{i} \sum_{w}+i \gamma-\sum_{w=0}^{i} L_{w}}^{\alpha} B_{0}^{\gamma} \\
& \quad=\left[B_{\beta-\sum_{w=0}^{i} L_{w}}^{\alpha+i \gamma-\sum_{w=0}^{i} L_{w}}, B_{0}^{\gamma}\right]+B_{0}^{\gamma} B_{\beta-\sum_{w=0}^{i} L_{w}}^{\alpha+i \gamma-\sum_{w=0}^{i} L_{w}} \\
& \quad=\sum_{L_{i+1} \geq 1} b_{L_{i+1}}\left(\beta-\sum_{w=0}^{i} L_{w}, \gamma\right) B_{\beta-\sum_{w=0}^{i+1} L_{w}}^{\alpha+(i+1) \gamma-\sum_{w=0}^{i+1} L_{w}}+B_{0}^{\gamma} B_{\beta-\sum_{w=0}^{i} L_{w}}^{\alpha+i \gamma-\sum_{w=0}^{i} L_{w}},
\end{aligned}
$$

is

$$
\begin{aligned}
= & \sum_{i=1}^{k}\left(\begin{array}{c}
k \\
i
\end{array}\right) \sum_{L_{1}, L_{2}, \ldots, L_{i+1} \geq 1} \prod_{j=1}^{i+1} b_{L_{j}}\left(\beta-\sum_{w=0}^{j-1} L_{w}, \gamma\right)\left(B_{0}^{\gamma}\right)^{k-i} B_{\beta-\sum_{w=0}^{i+1} L_{w}}^{\alpha+(i+1) \gamma-\sum_{w=0}^{i+1} L_{w}} \\
& +\sum_{i=1}^{k}\left(\begin{array}{c}
k \\
i
\end{array}\right) \sum_{L_{1}, L_{2}, \ldots, L_{i} \geq 1} \prod_{j=1}^{i} b_{L_{j}}\left(\beta-\sum_{w=0}^{j-1} L_{w}, \gamma\right)\left(B_{0}^{\gamma}\right)^{k+1-i} B_{\beta-\sum_{w=0}^{i} L_{w}}^{\alpha+i \gamma-\sum_{w=0}^{i} L_{w}} \\
& +\sum_{L_{1} \geq 1} b_{L_{1}}(\beta, \gamma)\left(B_{0}^{\gamma}\right)^{k} B_{\beta-L_{1}}^{\alpha+\gamma-L_{1}}+\left(B_{0}^{\gamma}\right)^{k+1} B_{\beta}^{\alpha}
\end{aligned}
$$

which upon replacing $i$ by $i-1$ in the first sum, with $i$ now ranging from 2 to $k+1$, is

$$
\begin{aligned}
= & \sum_{i=2}^{k+1}\left(\begin{array}{c}
k \\
i-1
\end{array}\right) \sum_{L_{1}, L_{2}, \ldots, L_{i} \geq 1} \prod_{j=1}^{i} b_{L_{j}}\left(\beta-\sum_{w=0}^{j-1} L_{w}, \gamma\right)\left(B_{0}^{\gamma}\right)^{k+1-i} B_{\beta-\sum_{w=0}^{i} L_{w}}^{\alpha+i \gamma-\sum_{w=0}^{i} L_{w}} \\
& +\sum_{i=1}^{k}\left(\begin{array}{c}
k \\
i
\end{array}\right) \sum_{L_{1}, L_{2}, \ldots, L_{i} \geq 1} \prod_{j=1}^{i} b_{L_{j}}\left(\beta-\sum_{w=0}^{j-1} L_{w}, \gamma\right)\left(B_{0}^{\gamma}\right)^{k+1-i} B_{\beta-\sum_{w=0}^{i} L_{w}}^{\alpha+i \gamma-\sum_{w=0}^{i} L_{w}} \\
& +\sum_{L_{1} \geq 1} b_{L_{1}}(\beta, \gamma)\left(B_{0}^{\gamma}\right)^{k} B_{\beta-L_{1}}^{\alpha+\gamma-L_{1}}+\left(B_{0}^{\gamma}\right)^{k+1} B_{\beta}^{\alpha} .
\end{aligned}
$$

Extracting the $i=k+1$ term from the first sum and including

$$
\sum_{L_{1} \geq 1} b_{L_{1}}(\beta, \gamma)\left(B_{0}^{\gamma}\right)^{k} B_{\beta-L_{1}}^{\alpha+\gamma-L_{1}}
$$

as its $i=1$ term, the above becomes

$$
\begin{aligned}
= & \sum_{L_{1}, L_{2}, \ldots, L_{k+1} \geq 1} \prod_{j=1}^{k+1} b_{L_{j}}\left(\beta-\sum_{w=0}^{j-1} L_{w}, \gamma\right) B_{\beta-\sum_{w=0}^{k+1} L_{w}}^{\alpha+(k+1) \gamma-\sum_{w=0}^{k+1} L_{w}} \\
& +\sum_{i=1}^{k}\left(\left(\begin{array}{c}
k \\
i-1
\end{array}\right)+\left(\begin{array}{c}
k \\
i
\end{array}\right)\right) \times \sum_{L_{1}, L_{2}, \ldots, L_{i} \geq 1} \prod_{j=1}^{i} b_{L_{j}}\left(\beta-\sum_{w=0}^{j-1} L_{w}, \gamma\right) \\
& \times\left(B_{0}^{\gamma}\right)^{k+1-i} B_{\beta-\sum_{w=0}^{i} L_{w}}^{\alpha+i \gamma-\sum_{w=0}^{i} L_{w}}+\left(B_{0}^{\gamma}\right)^{k+1} B_{\beta}^{\alpha}
\end{aligned}
$$


which, since

$$
\left(\begin{array}{c}
k \\
i-1
\end{array}\right)+\left(\begin{array}{c}
k \\
i
\end{array}\right)=\left(\begin{array}{c}
k+1 \\
i
\end{array}\right)
$$

is

$$
\begin{aligned}
& =\sum_{i=1}^{k+1}\left(\begin{array}{c}
k+1 \\
i
\end{array}\right) \sum_{L_{1}, L_{2}, \ldots, L_{i} \geq 1} \prod_{j=1}^{i} b_{L_{j}}\left(\beta-\sum_{w=0}^{j-1} L_{w}, \gamma\right) \\
& \times\left(B_{0}^{\gamma}\right)^{k+1-i} B_{\beta-\sum_{w=0}^{i} L_{w}+i \gamma-\sum_{w=0}^{i} L_{w}}^{\alpha+}+\left(B_{0}^{\gamma}\right)^{k+1} B_{\beta}^{\alpha} .
\end{aligned}
$$

\section{Non-Existence of a Fock Representation of the Renormalized Oscillator Algebras}

Definition 4.1. A representation of the RPQWN Lie algebra on a Hilbert space $\mathcal{H}$ is called Fock if there exists a unit vector $\Phi \in \mathcal{H}$, called "vacuum vector", such that:

$$
\begin{array}{ll}
B_{k}^{0} \Phi=0, & \forall k \in \mathbb{N}, \\
B_{k}^{h} \Phi=0, & \forall k>0, h \geq 0 .
\end{array}
$$

The set of vectors

$$
\mathcal{N}:=\left\{\left(B_{0}^{k_{N}}\right)^{n_{N}} \cdots\left(B_{0}^{k_{1}}\right)^{n_{1}} \Phi: N \in \mathbb{N}, n_{1}, \ldots, n_{N}, k_{1}, \ldots, k_{N} \in \mathbb{N}\right\}
$$

is total in $\mathcal{H}$ and

$$
\left\langle B_{0}^{k} \xi, \eta\right\rangle=\left\langle\xi, B_{k}^{0} \eta\right\rangle ; \quad \forall k \geq 0 \quad \forall \xi, \eta \in \mathcal{N} .
$$

If $\mathcal{H}$ is a vector space and $\Phi \in \mathcal{H}$ is a unit vector satisfying (4.2) and (4.3), then on the algebraic linear span of $\mathcal{N}$ there exists one and only one sesquilinear form $\langle\cdot, \cdot\rangle$ satisfying (4.4). It is not difficult to verify that, if a Fock representation exists, it is unique up to isomorphism.

Do there exist Fock representations of the RPQWN Lie algebra? The answer depends on the choice of the renormalization constants $c_{n}$. The following theorem shows that, with a single renormalization constant, i.e. with $c_{l-1}=c^{l-1}$ (cf. (3.8)), no Fock representation can exist if, for some $n \geq 1, B_{0}^{n}$ and $B_{0}^{2 n}$ have the correlations deduced from the commutation relations (3.6) and the Fock prescriptions (4.1), (4.2).

Corollary 4.1. Suppose that the Fock representation exists, then for all $\alpha, \beta, \gamma \geq 0$ and all $n \geq 1$ one has:

$$
\begin{aligned}
B_{\beta}^{\alpha}\left(B_{0}^{\gamma}\right)^{n} \Phi= & \delta_{\beta, 0}\left(B_{0}^{\gamma}\right)^{n} B_{0}^{\alpha} \Phi+\sum_{i=1}^{n}\left(\begin{array}{c}
n \\
i
\end{array}\right) \sum_{\substack{L_{1}, L_{2}, \ldots, L_{i} \geq 1 \\
L_{1}+L_{2}+\cdots+L_{i}=\beta}} \prod_{j=1}^{i} b_{L_{j}}\left(\beta-\sum_{w=0}^{j-1} L_{w}, \gamma\right) \\
& \times\left(B_{0}^{\gamma}\right)^{n-i} B_{0}^{\alpha+i \gamma-\beta} \Phi,
\end{aligned}
$$

where $L_{0}=0$. 
Proof. Follows directly from Proposition 3.1 and the fact that

$$
B_{\beta}^{\alpha} \Phi=\delta_{\beta, 0} B_{0}^{\alpha} \Phi
$$

Theorem 4.1. Let $\mathcal{L}$ be a Lie *-sub-algebra of the $R P Q W N$ Lie algebra with the following properties:

(i) $\mathcal{L}$ contains $B_{0}^{n}$, and $B_{0}^{2 n}$ where, in the notation (3.9) the noise operators are defined on the same interval $I$ and $B_{0}^{0}\left(\chi_{I}\right)=\mu(I)$.

(ii) the $B_{K}^{N}$ satisfy the commutation relations (3.6) with $c_{l-1}$ satisfying (3.1) and (3.8).

Then $\mathcal{L}$ has no Fock representation if the interval I is such that

$$
\mu(I)<\frac{1}{c}
$$

Proof. By contradiction. If a Fock representation exists, then for any $a, b \in \mathbb{R}$ one must have:

$$
\left\langle\left(a B_{0}^{2 n}+b\left(B_{0}^{n}\right)^{2}\right) \Phi,\left(a B_{0}^{2 n}+b\left(B_{0}^{n}\right)^{2}\right) \Phi\right\rangle=\left\|a B_{0}^{2 n} \Phi+b\left(B_{0}^{n}\right)^{2} \Phi\right\|^{2} \geq 0 .
$$

Using the notation $\langle x\rangle=\langle\Phi, x \Phi\rangle$, and since, under our assumptions $\left\langle B_{2 n}^{0}\left(B_{0}^{n}\right)^{2}\right\rangle=\left\langle\left(B_{n}^{0}\right)^{2} B_{0}^{2 n}\right\rangle$, this amounts to the positive semi-definiteness of the quadratic form

$$
a^{2}\left\langle B_{2 n}^{0} B_{0}^{2 n}\right\rangle+2 a b\left\langle B_{2 n}^{0}\left(B_{0}^{n}\right)^{2}\right\rangle+b^{2}\left\langle\left(B_{n}^{0}\right)^{2}\left(B_{0}^{n}\right)^{2}\right\rangle
$$

or

$$
u A u^{t} \geq 0
$$

where

$$
u=\left(\begin{array}{ll}
a & b
\end{array}\right), \quad u^{t}=\left(\begin{array}{l}
a \\
b
\end{array}\right)
$$

and $A$ is the symmetric real matrix

$$
A=\left(\begin{array}{cc}
\left\langle B_{2 n}^{0} B_{0}^{2 n}\right\rangle & \left\langle B_{2 n}^{0}\left(B_{0}^{n}\right)^{2}\right\rangle \\
\left\langle B_{2 n}^{0}\left(B_{0}^{n}\right)^{2}\right\rangle & \left\langle\left(B_{n}^{0}\right)^{2}\left(B_{0}^{n}\right)^{2}\right\rangle
\end{array}\right) .
$$

Using Proposition 3.1 and Corollary 4.1 we have

$$
\begin{aligned}
B_{2 n}^{0} B_{0}^{2 n} \Phi=\left(\left[B_{2 n}^{0}, B_{0}^{2 n}\right]+B_{0}^{2 n} B_{2 n}^{0}\right) \Phi=\left[B_{2 n}^{0}, B_{0}^{2 n}\right] \Phi & =(2 n)^{(2 n)} c^{2 n-1} B_{0}^{0} \Phi \\
& =(2 n) ! c^{2 n-1} \mu(I) \Phi .
\end{aligned}
$$

Thus

$$
\left\langle B_{2 n}^{0} B_{0}^{2 n}\right\rangle=(2 n) ! c^{2 n-1} \mu(I) .
$$


Similarly,

$$
\begin{aligned}
B_{2 n}^{0}\left(B_{0}^{n}\right)^{2} \Phi= & \sum_{i=1}^{2}\left(\begin{array}{c}
2 \\
i
\end{array}\right) \sum_{\substack{L_{1}, \ldots, L_{i} \geq 1 \\
L_{1}+\cdots+L_{i}=2 n}} \prod_{j=1}^{n} b_{L_{j}}\left(2 n-\sum_{w=0}^{j-1} L_{w}, n\right)\left(B_{0}^{n}\right)^{2-i} B_{0}^{i n-2 n} \Phi \\
= & 2 b_{2 n}(2 n, n)\left(B_{0}^{n}\right)^{1} B_{0}^{-n} \Phi+\sum_{\substack{L_{1}, L_{2} \geq 1 \\
L_{1}+L_{2}=2 n}} b_{L_{1}}(2 n, n) b_{L_{2}}\left(2 n-L_{1}, n\right) B_{0}^{0} \Phi \\
= & 0+\sum_{\substack{L_{1}, L_{2} \geq 1 \\
L_{1}+L_{2}=2 n}}\left(\begin{array}{c}
2 n \\
L_{1}
\end{array}\right) n^{\left(L_{1}\right)} c^{L_{1}-1}\left(\begin{array}{c}
2 n-L_{1} \\
L_{2}
\end{array}\right) n^{\left(L_{2}\right)} c^{L_{2}-1} \mu(I) \Phi \\
= & \sum_{\substack{L_{1}, L_{2} \geq 1 \\
L_{1}+L_{2}=2 n}}\left(\begin{array}{c}
2 n \\
L_{1}
\end{array}\right)\left(\begin{array}{c}
2 n-L_{1} \\
L_{2}
\end{array}\right) n^{\left(L_{1}\right)} n^{\left(L_{2}\right)} c^{L_{1}+L_{2}-2} \mu(I) \Phi \\
= & \left(\begin{array}{c}
2 n \\
n
\end{array}\right)\left(\begin{array}{c}
2 n-n \\
n
\end{array}\right) n^{(n)} n^{(n)} c^{n+n-2} \mu(I) \Phi \\
= & (2 n) ! c^{2 n-2} \mu(I) \Phi
\end{aligned}
$$

since only for $L_{1}=L_{2}=n$ both factorial powers are nonzero. Thus

$$
\left\langle B_{2 n}^{0}\left(B_{0}^{n}\right)^{2}\right\rangle=(2 n) ! c^{2 n-2} \mu(I) .
$$

Finally,

$$
\begin{aligned}
B_{n}^{0}\left(B_{0}^{n}\right)^{2} \Phi= & \sum_{i=1}^{2}\left(\begin{array}{l}
2 \\
i
\end{array}\right) \sum_{\substack{L_{1}, \ldots, L_{i} \geq 1 \\
L_{1}+\cdots+L_{i}=2 n}} \prod_{j=1}^{n} b_{L_{j}}\left(n-\sum_{w=0}^{j-1} L_{w}, n\right)\left(B_{0}^{n}\right)^{2-i} B_{0}^{i n-n} \Phi \\
= & 2 b_{n}(n, n) B_{0}^{n} B_{0}^{0} \Phi+\sum_{\substack{L_{1}, L_{2} \geq 1 \\
L_{1}+L_{2}=n}} b_{L_{1}}(n, n) b_{L_{2}}\left(n-L_{1}, n\right) B_{0}^{n} \Phi \\
= & 2 b_{n}(n, n) \mu(I) B_{0}^{n} \Phi+\sum_{L_{1}=1}^{n-1} b_{L_{1}}(n, n) b_{n-L_{1}}\left(n-L_{1}, n\right) B_{0}^{n} \Phi \\
= & 2\left(\begin{array}{c}
n \\
n
\end{array}\right) n^{(n)} c^{n-1} \mu(I) B_{0}^{n} \Phi+\sum_{L_{1}=1}^{n-1}\left(\begin{array}{c}
n \\
L_{1}
\end{array}\right) n^{\left(L_{1}\right)} c^{L_{1}-1}\left(\begin{array}{c}
n-L_{1} \\
n-L_{1}
\end{array}\right) \\
& \times n^{\left(n-L_{1}\right)} c^{n-L_{1}-1} B_{0}^{n} \Phi \\
= & 2(n !) c^{n-1} \mu(I) B_{0}^{n} \Phi+\sum_{L_{1}=1}^{n-1}\left(\begin{array}{c}
n \\
L_{1}
\end{array}\right) n^{\left(L_{1}\right)} n^{\left(n-L_{1}\right)} c^{n-2} B_{0}^{n} \Phi \\
= & 2(n !) c^{n-1} \mu(I) B_{0}^{n} \Phi+\left((2 n)^{(n)}-2(n !)\right) c^{n-2} B_{0}^{n} \Phi
\end{aligned}
$$


since, by the binomial theorem for factorial powers

$$
(x+y)^{(m)}=\sum_{k=0}^{m}\left(\begin{array}{c}
m \\
k
\end{array}\right) x^{(m-k)} y^{(k)}
$$

we have

$$
\begin{aligned}
\sum_{L_{1}=1}^{n-1}\left(\begin{array}{c}
n \\
L_{1}
\end{array}\right) n^{\left(L_{1}\right)} n^{\left(n-L_{1}\right)} & =(n+n)^{(n)}-\left(\begin{array}{c}
n \\
n
\end{array}\right) n^{(n)} n^{(0)}-\left(\begin{array}{c}
n \\
0
\end{array}\right) n^{(0)} n^{(n)} \\
& =(2 n)^{(n)}-2(n !) .
\end{aligned}
$$

Thus

$$
\left(B_{n}^{0}\right)^{2}\left(B_{0}^{n}\right)^{2} \Phi=2(n !) c^{n-1} \mu(I) B_{n}^{0} B_{0}^{n} \Phi+\left((2 n)^{(n)}-2(n !)\right) c^{n-2} B_{n}^{0} B_{0}^{n} \Phi
$$

and since

$$
B_{n}^{0} B_{0}^{n} \Phi=\left(\left[B_{n}^{0}, B_{0}^{n}\right]+B_{0}^{n} B_{n}^{0}\right) \Phi=\left[B_{n}^{0}, B_{0}^{n}\right] \Phi=(n)^{(n)} c^{n-1} B_{0}^{0} \Phi=n ! c^{n-1} \mu(I) \Phi
$$

we obtain

$$
\left(B_{n}^{0}\right)^{2}\left(B_{0}^{n}\right)^{2} \Phi=2(n !)^{2} c^{2 n-2} \mu(I)^{2} \Phi+\left((2 n) !-2(n !)^{2}\right) c^{2 n-3} \mu(I) \Phi
$$

and so

$$
\left\langle\left(B_{n}^{0}\right)^{2}\left(B_{0}^{n}\right)^{2}\right\rangle=2(n !)^{2} c^{2 n-2} \mu(I)^{2}+\left((2 n) !-2(n !)^{2}\right) c^{2 n-3} \mu(I) .
$$

Thus

$$
A=\left(\begin{array}{cc}
(2 n) ! c^{2 n-1} \mu(I) & (2 n) ! c^{2 n-2} \mu(I) \\
(2 n) ! c^{2 n-2} \mu(I) & 2(n !)^{2} c^{2 n-2} \mu(I)^{2}+\left((2 n) !-2(n !)^{2}\right) c^{2 n-3} \mu(I)
\end{array}\right) .
$$

$A$ is a symmetric matrix, so it is positive semi-definite if and only if its minors are non-negative. The minor determinants of $A$ are

$$
d_{1}=(2 n) ! c^{2 n-1} \mu(I) \geq 0
$$

and

$$
d_{2}=2 c^{4(n-1)} \mu(I)^{2}(n !)^{2}(2 n) !(c \mu(I)-1) \geq 0 \Leftrightarrow \mu(I) \geq \frac{1}{c} .
$$

Theorem 4.2. Let $\mathcal{L}$ be a Lie *-sub-algebra of the $R P Q W N$ Lie algebra with the following properties:

For any $k>n \geq 1$

(i) $\mathcal{L}$ contains $B_{0}^{n}, B_{0}^{k}$, and $B_{0}^{n+k}$ where, in the notation (3.9) the noise operators are defined on the same interval $I$ and $B_{0}^{0}\left(\chi_{I}\right)=\mu(I)$,

(ii) the $B_{K}^{N}$ satisfy the commutation relations (3.6) with $c_{l-1}$ satisfying (3.1) and (3.8).

Then $\mathcal{L}$ does not have a Fock representation if the interval $I$ is such that

$$
\mu(I)<\frac{r}{c}
$$


where

$$
r=\frac{(n+k) !-\left((n+k)^{(n)}-k^{(n)}-n !\right) k !}{n ! k !}>0, \quad \forall n, k \in \mathbb{N} \backslash\{0\} .
$$

Proof. (By contradiction) If a Fock representation exists, then for any $a, b \in \mathbb{R}$ one must have:

$$
\left\langle\left(a B_{0}^{n+k}+b B_{0}^{n} B_{0}^{k}\right) \Phi,\left(a B_{0}^{n+k}+b B_{0}^{n} B_{0}^{k}\right) \Phi\right\rangle=\left\|\left(a B_{0}^{n+k}+b B_{0}^{n} B_{0}^{k}\right) \Phi\right\|^{2} \geq 0 .
$$

Using the notation $\langle x\rangle=\langle\Phi, x \Phi\rangle$ this amounts to the positive semi-definiteness of the quadratic form

$$
a^{2}\left\langle B_{n+k}^{0} B_{0}^{n+k}\right\rangle+2 a b\left\langle B_{n+k}^{0} B_{0}^{n} B_{0}^{k}\right\rangle+b^{2}\left\langle B_{k}^{0} B_{n}^{0} B_{0}^{n} B_{0}^{k}\right\rangle
$$

or

$$
u A u^{t} \geq 0
$$

where

$$
u=\left(\begin{array}{ll}
a & b
\end{array}\right), \quad u^{t}=\left(\begin{array}{l}
a \\
b
\end{array}\right)
$$

and $A$ is the symmetric real matrix

$$
A=\left(\begin{array}{cc}
\left\langle B_{n+k}^{0} B_{0}^{n+k}\right\rangle & \left\langle B_{n+k}^{0} B_{0}^{n} B_{0}^{k}\right\rangle \\
\left\langle B_{n+k}^{0} B_{0}^{n} B_{0}^{k}\right\rangle & \left\langle B_{k}^{0} B_{n}^{0} B_{0}^{n} B_{0}^{k}\right\rangle
\end{array}\right) .
$$

We have

$$
\begin{aligned}
B_{n+k}^{0} B_{0}^{n+k} \Phi & =\left(\left[B_{n+k}^{0}, B_{0}^{n+k}\right]+B_{0}^{n+k} B_{n+k}^{0}\right) \Phi=\left[B_{n+k}^{0}, B_{0}^{n+k}\right] \Phi \\
& =(n+k)^{(n+k)} c^{n+k-1} B_{0}^{0} \Phi=(n+k) ! c^{n+k-1} \mu(I) \Phi .
\end{aligned}
$$

Thus

$$
\left\langle B_{n+k}^{0} B_{0}^{n+k}\right\rangle=(n+k) ! c^{n+k-1} \mu(I) .
$$

Similarly,

$$
\begin{aligned}
\left\langle B_{n+k}^{0} B_{0}^{n} B_{0}^{k}\right\rangle & =\left\langle B_{n}^{0} B_{0}^{n+k} \Phi, B_{0}^{k} \Phi\right\rangle=\left\langle\left[B_{n}^{0} B_{0}^{n+k}\right] \Phi, B_{0}^{k} \Phi\right\rangle \\
& =b_{n}(n, n+k)\left\langle B_{k}^{0} B_{0}^{k}\right\rangle=b_{n}(n, n+k) b_{k}(k, k) \mu(I) \\
& =(n+k) ! c^{n+k-2} \mu(I) .
\end{aligned}
$$

Finally,

$$
\begin{aligned}
B_{n}^{0} B_{0}^{n} & B_{0}^{k} \Phi \\
= & \left(\left[B_{n}^{0}, B_{0}^{n}\right]+B_{0}^{n} B_{n}^{0}\right) B_{0}^{k} \Phi=\left[B_{n}^{0}, B_{0}^{n}\right] B_{0}^{k} \Phi+B_{0}^{n}\left[B_{n}^{0} B_{0}^{k}\right] \Phi \\
= & \sum_{L \geq 1} b_{L}(n, n)\left(\left[B_{n-L}^{n-L}, B_{0}^{k}\right]+B_{0}^{k} B_{n-L}^{n-L}\right) \Phi+B_{0}^{n} \sum_{L^{\prime} \geq 1} b_{L^{\prime}}(n, k) B_{n-L^{\prime}}^{k-L^{\prime}} \Phi \\
& =\sum_{L=1}^{n-1} b_{L}(n, n) b_{n-L}(n-L, k) B_{0}^{k} \Phi+n ! c^{n-1} \mu(I) B_{0}^{k} \Phi+k^{(n)} c^{n-1} B_{0}^{n} B_{0}^{k-n} \Phi
\end{aligned}
$$


and, using the binomial theorem for factorial powers, we obtain

$$
\begin{aligned}
B_{n}^{0} B_{0}^{n} B_{0}^{k} \Phi= & \left((n+k)^{(n)}-k^{(n)}-n !\right) c^{n-2} B_{0}^{k} \Phi+n ! c^{n-1} \mu(I) B_{0}^{k} \Phi \\
& +k^{(n)} c^{n-1} B_{0}^{n} B_{0}^{k-n} \Phi
\end{aligned}
$$

and so

$$
\begin{aligned}
B_{k}^{0} B_{n}^{0} B_{0}^{n} B_{0}^{k} \Phi= & \left((n+k)^{(n)}-k^{(n)}-n !\right) c^{n-2} B_{k}^{0} B_{0}^{k} \Phi+n ! c^{n-1} \mu(I) B_{k}^{0} B_{0}^{k} \Phi \\
& +k^{(n)} c^{n-1} B_{k}^{0} B_{0}^{n} B_{0}^{k-n} \Phi .
\end{aligned}
$$

Using

$$
\left\langle B_{k}^{0} B_{0}^{k}\right\rangle=k ! c^{k-1} \mu(I)
$$

and

$$
\left\langle B_{k}^{0} B_{0}^{n} B_{0}^{k-n}\right\rangle=k^{(n)}(k-n) ! c^{k-2} \mu(I)
$$

we obtain

$$
\begin{aligned}
\left\langle B_{k}^{0} B_{n}^{0} B_{0}^{n} B_{0}^{k}\right\rangle= & \left(\left((n+k)^{(n)}-k^{(n)}-n !\right) k !+\left(k^{(n)}\right)^{2}(k-n) !\right) c^{n+k-3} \mu(I) \\
& +n ! k ! c^{n+k-2} \mu(I)^{2}
\end{aligned}
$$

Therefore

$$
A=\left(\begin{array}{cc}
(n+k) ! c^{n+k-1} \mu(I) & (n+k) ! c^{n+k-2} \mu(I) \\
& \left(\left((n+k)^{(n)}-k^{(n)}-n !\right) k !\right. \\
(n+k) ! c^{n+k-2} \mu(I) & \left.+\left(k^{(n)}\right)^{2}(k-n) !\right) c^{n+k-3} \mu(I) \\
& +n ! k ! c^{n+k-2} \mu(I)^{2}
\end{array}\right)
$$

$A$ is a symmetric matrix, so it is positive semi-definite if and only if its minors are non-negative. The minor determinants of $A$ are

$$
d_{1}=(n+k) ! c^{n+k-1} \mu(I) \geq 0
$$

and

$$
d_{2}=\mu(I)^{2}(n+k) ! c^{2 n+2 k-4}\left(\left((n+k)^{(n)}-k^{(n)}-n !\right) k !-(n+k) !+n ! k ! c \mu(I)\right)
$$

which is $\geq 0 \Leftrightarrow \mu(I) \geq \frac{r}{c}$.

Theorem 4.3. Any *-Lie algebra that contains $B_{3}^{0}$ and $B_{0}^{3}$ also contains $B_{6}^{0}$ and $B_{0}^{6}$, hence it does not admit a Fock representation.

Proof. We have, in the notations of the introduction and with $N:=a^{\dagger} a$,

$$
\left[a^{3}, a^{\dagger^{3}}\right]=9 N^{2}+18 N+6 \in \mathcal{L},
$$

where $\mathcal{L}$ is the Lie algebra generated by $a^{3}, a^{\dagger^{3}}$. Therefore

$$
\left[a^{3}, 9 N^{2}+18 N+6\right]=108 a^{3}+54 N a^{3} \in \mathcal{L} .
$$


Since $a^{3} \in \mathcal{L}$, also $N a^{3} \in \mathcal{L}$. But then

$$
\left[a^{3}, N a^{3}\right]=3 a^{6} \in \mathcal{L}
$$

which implies that $a^{6} \in \mathcal{L}$. Since the Lie algebra generated by $B_{3}^{0}$ and $B_{0}^{3}$ is isomorphic to the one generated by $a^{3}$ and $a^{\dagger^{3}}$, it will contain $B_{6}^{0}$ and $B_{0}^{6}$. Therefore the proof follows from Theorem 4.1 .

\section{Non-Existence of Gaussian Representations}

Theorem 5.1. Let $\mathcal{A}$ be a *-algebra of operator valued distributions on $\mathbb{R}^{d}$ whose test function space includes the characteristic functions of intervals in $\mathbb{R}^{d}$ defined as follows:

$$
(a, b)=\left\{\begin{array}{l}
\left\{x=\left(x_{j}\right): a_{j}<x_{j}<b_{j}, \quad j=1, \ldots, d\right\}, \quad \text { if } a_{j}<b_{j}, \forall j \\
\phi, \text { if } a_{j}>b_{j} \text { for some } j
\end{array}\right.
$$

and let

$$
b_{k}^{2}, b_{k}^{+2}, b_{h}, b_{h}^{+}
$$

be elements of $\mathcal{A}$. There exists no state $\langle\cdot\rangle$ on $\mathcal{A}$ with the following properties:

$$
\begin{aligned}
\left\langle b_{k} b_{h}^{+}\right\rangle & =\mu(k) \delta(k-h), \\
\left\langle b_{k^{\prime}} b_{k} b_{h^{\prime}}^{+} b_{h}^{+}\right\rangle & =2 \mu(k) \mu\left(k^{\prime}\right) \delta\left(k^{\prime}-h^{\prime}\right) \delta(k-h), \\
\left\langle b_{k}^{2} b_{h^{\prime}}^{+} b_{h}^{+}\right\rangle & =2 \mu(k)^{2} \delta\left(k-h^{\prime}\right) \delta(k-h), \\
\left\langle b_{k}^{2} b_{h}^{+2}\right\rangle & =\sigma(k) \delta(k-h),
\end{aligned}
$$

where $\sigma \in L_{\mathrm{loc}}^{1}\left(\mathbb{R}^{d}\right)$ and $\mu \in L_{\mathrm{loc}}^{1} \cap L_{\mathrm{loc}}^{2}(\mathbb{R})$ are such that there exist an interval $I \subseteq \mathbb{R}^{d}$ and constants $M_{I}, \varepsilon_{I}>0$ such that

$$
+\infty>M_{I}>\sigma(k), \quad \mu(k) ; \quad \mu(k) \geq \varepsilon_{I}>0, \quad \forall k \in I .
$$

Remark. (5.4) is deduced from the usual (boson) Gaussian rule with mean zero and covariance given by (5.3). (5.5) is obtained from (5.4) by putting (formally) $k^{\prime}=$ $k$. (5.6) is obtained from (5.5) by putting (formally) $h^{\prime}=h$ and renormalizing $\delta^{2}(k-$ $h$ ), i.e. replacing $2 \mu(k)^{2}$ by the renormalizing factor $\sigma(k)$. Since no assumptions have been made on the remaining correlations, the above considerations prove the nonexistence of a state with the following properties: (i) it is boson Gaussian when restricted to the first-order noise (cf. (5.3) and (5.4)); (ii) it extends the usual Gaussian rule to fourth moments involving $b_{k}^{2}$ and $b_{k}^{+2}$ (cf. (5.5) and (5.6)). Finally, since $I$ is an arbitrary bounded interval, for any $\beta>0$ we can always find an $I$ such that (5.7) is satisfied if $I$ is an interval not containing 0 in its closure and such that

$$
\mu(k)=\frac{1}{e^{\beta \omega_{k}}-1}, \quad \sigma(k)=\frac{c}{\left(e^{\beta \omega_{k}}-1\right)^{2}},
$$


where $c>0$ is a (renormalization) constant and $\omega$ is a function satisfying the conditions described in Sec. 5.8 of Ref. 4 . Since the above choice of $\mu(k)$ characterizes the equilibrium boson Gaussian states at inverse temperature $\beta$ (cf. Sec. 2.10 of Ref. 4), this implies that the above result extends to the finite temperature (and more general) cases the no-go theorems proved in Ref. 11 and Ref. 3.

Proof. If such a state exists, then the matrix

$$
\begin{aligned}
& \left(\begin{array}{ll}
\left\langle b_{k}^{2} b_{h}^{+2}\right\rangle & \left\langle b_{k}^{2} b_{h^{\prime}}^{+} b_{h^{\prime \prime}}^{+}\right\rangle \\
\left\langle b_{h^{\prime \prime}} b_{h^{\prime}} b_{k}^{+2}\right\rangle & \left\langle b_{k^{\prime}} b_{k^{\prime \prime}} b_{h^{\prime}}^{+} b_{h^{\prime \prime}}^{+}\right\rangle
\end{array}\right) \\
& \quad=\left(\begin{array}{ll}
\sigma(k) \delta(k-h) & \mu(k)^{2} 2 \delta\left(k-h^{\prime}\right) \delta\left(k-h^{\prime \prime}\right) \\
2 \mu(k)^{2} \delta\left(k-h^{\prime}\right) \delta\left(k-h^{\prime \prime}\right) \mu\left(k^{\prime \prime}\right) \mu\left(k^{\prime}\right) & 2 \mu\left(k^{\prime}\right) \mu\left(k^{\prime \prime}\right) \delta\left(k^{\prime}-h^{\prime}\right) \delta\left(k^{\prime \prime}-h^{\prime \prime}\right)
\end{array}\right)
\end{aligned}
$$

must be positive definite, expressing the positivity of the expectation value

$$
\left\langle\left|\alpha b_{h}^{+2}+\beta b_{h^{\prime}}^{+} b_{h^{\prime \prime}}^{+}\right|^{2}\right\rangle
$$

for any $\alpha, \beta \in \mathbb{C}$. In the notations (3.3)-(3.5) the positivity of (5.8) is equivalent to the positivity of the covariance matrix

$$
\begin{aligned}
& \left(\begin{array}{cc}
\left\langle B_{2}^{0}(\varphi) B_{0}^{2}(\varphi)\right\rangle & \left\langle B_{2}^{0}(\varphi) B_{0}^{1}(\xi)^{2}\right\rangle \\
\left\langle B_{1}^{0}(\xi)^{2} B_{0}^{2}(\varphi)\right\rangle & \left\langle B_{1}^{0}(\xi)^{2} B_{0}^{1}(\xi)^{2}\right\rangle
\end{array}\right) \\
& \quad=\left(\begin{array}{cc}
\int|\varphi(k)|^{2} \sigma(k) d k & 2 \int \bar{\varphi}(k)^{2} \xi(k)^{2} d k \mu(k)^{2} \\
2 \int \varphi(k)^{2} \bar{\xi}(k)^{2} \mu(k)^{2} d k & 2\left(\int|\xi(k)|^{2} \mu(k) d k\right)^{2}
\end{array}\right)
\end{aligned}
$$

for any choice of test functions $\varphi, \xi$ on $\mathbb{R}^{d}$. Taking

$$
\varphi(k)=\xi(k)=\chi_{I}(k),
$$

where $I \subseteq \mathbb{R}^{d}$ is any interval, one finds

$$
\left(\begin{array}{ll}
\int_{I} \sigma(k) d k & 2 \int_{I} \mu(k)^{2} d k \\
2 \int_{I} \mu(k)^{2} d k & 2\left(\int_{I} \mu(k) d k\right)^{2}
\end{array}\right)=:\left(\begin{array}{ll}
\sigma(I) & 2 \mu^{2}(I) \\
2 \mu^{2}(I) & 2(\mu(I))^{2}
\end{array}\right)=: F(I) .
$$

Now let us choose $I$ so that condition (5.7) is satisfied and let $I_{n+1} \subseteq I_{n} \subseteq I$ be a sequence of bounded open intervals such that

$$
\bigcap_{n} I_{n}=\emptyset .
$$


Then, for $\alpha=1,2$, because of assumption (5.7), one has:

$$
\begin{aligned}
\limsup _{n \rightarrow \infty} \frac{\mu\left(I_{n}\right)}{\left|I_{n}\right|}=\limsup _{n \rightarrow \infty} \frac{1}{\left|I_{n}\right|} \int_{I_{n}} \mu(k) d k \leq M_{I}<\infty, \\
\liminf _{n \rightarrow \infty} \frac{\mu^{2}\left(I_{n}\right)}{\left|I_{n}\right|}=\liminf _{n \rightarrow \infty} \frac{1}{\left|I_{n}\right|} \int_{I_{n}} \mu(k)^{2} d k \geq \varepsilon_{I}^{2}>0, \\
\lim _{n \rightarrow \infty} \frac{\sigma\left(I_{n}\right)}{\left|I_{n}\right|}=\lim _{n \rightarrow \infty} \int_{I_{n}} \sigma(k) d k=0 .
\end{aligned}
$$

Therefore, denoting

$$
D(I):=\operatorname{det} F(I)=2 \sigma(I)(\mu(I))^{2}-4\left(\mu^{2}(I)\right)^{2}
$$

we have

$$
\limsup _{n \rightarrow \infty} \frac{D\left(I_{n}\right)}{\left|I_{n}\right|^{2}} \leq-4 \varepsilon_{I}^{4}<0 .
$$

Thus, if $n$ is large enough (5.9)-(5.11) imply that:

$$
D\left(I_{n}\right) \leq-4 \varepsilon_{I}^{4}\left|I_{n}\right|^{2}<0
$$

and the matrix (5.8) cannot be positive definite.

Corollary 5.1. Let $\mathcal{T}$ be any complex vector space of complex valued functions on $\mathbb{R}^{d}$ containing the characteristic functions of all open intervals (cf. (5.1)) and let $\sigma$ and $\mu$ be as in Theorem 5.1. With the notations $($ for $\psi \in \mathcal{T})$

$$
\sigma(\psi):=\int_{\mathbb{R}^{d}} \psi(k) \sigma(k) d k, \quad \mu(\psi):=\int_{\mathbb{R}^{d}} \psi(k) \mu(k) d k, \quad \mu^{2}(\psi):=\int_{\mathbb{R}^{d}} \psi(k) \mu(k)^{2} d k
$$

there exists no algebraic probability space $\{\mathcal{A},\langle\cdot\rangle\}$ and linear maps

$$
B_{2}^{+}, B_{1}^{+}: \psi \in \mathcal{T} \rightarrow B_{2}^{+}(\varphi), \quad B_{1}^{+}(\varphi) \in \mathcal{A}
$$

such that, denoting $B_{j}:=\left(B_{j}^{+}\right)^{*}$, one has

$$
\left(\begin{array}{cc}
\left\langle B_{2}(\varphi) B_{2}^{+}(\varphi)\right\rangle & \left\langle B_{2}(\varphi)\left(B_{1}^{+}(\xi)\right)^{2}\right\rangle \\
\left\langle B_{1}(\xi)^{2} B_{2}^{+}(\varphi)\right\rangle & \left\langle B_{1}(\xi)^{2} B_{1}^{+}(\xi)^{2}\right\rangle
\end{array}\right)=\left(\begin{array}{ll}
\sigma\left(|\varphi|^{2}\right) & 2 \mu^{2}\left(\bar{\varphi}^{2} \xi^{2}\right) \\
2 \mu^{2}\left(\varphi^{2} \bar{\xi}^{2}\right) & 2\left(\mu\left(|\xi|^{2}\right)\right)^{2}
\end{array}\right) .
$$

Proof. This is simply a rephrasing of Theorem 4.2 for smeared fields.

\section{Acknowledgments}

A. B. wishes to thank Professor Luigi Accardi for the hospitality of the Centro Vito Volterra of the Universita di Roma Tor Vergata where this work was completed. 


\section{References}

1. L. Accardi, Meixner classes and the square of white noise, talk given at $A M S-A M A$ Joint Mathematics Meetings, New Orleans, LA, January 10-13, 2001, AMS Contemporary Mathematics, Vol. 317, eds. H.-H. Kuo and A. Sengupta (Amer. Math. Soc., 2003), pp. 1-13.

2. L. Accardi and A. Boukas, The unitarity conditions for the square of white noise, Inf. Dim. Anal. Quantum Probab. Rel. Topics 6 (2003) 1-26.

3. L. Accardi, U. Franz and M. Skeide, Renormalized squares of white noise and nonGaussian noises as Levy processes on real Lie algebras, Commun. Math. Phys. 228 (2002) 123-150.

4. L. Accardi, Y. G. Lu and I. V. Volovich, Quantum Theory and Its Stochastic Limit (Springer, 2002).

5. L. Accardi, Y. G. Lu and I. V. Volovich, White noise approach to classical and quantum stochastic calculi, Trento, Italy, 1999, Volterra Center preprint 375.

6. L. Accardi and N. Obata, Towards a nonlinear extension of stochastic calculus, Publications of the Research Institute for Mathematical Sciences, Kyoto, RIMS Kokyuroku 957, ed. N. Obata (RIMS, 1996), pp. 1-15.

7. L. Accardi, G. Amosov and U. Franz, Second quantized automorphisms of the renormalized square of white noise (RSWN) algebra, Inf. Dim. Anal. Quantum Probab. Rel. Topics 7 (2004) 183-194.

8. L. Accardi and A. Boukas, White noise calculus and stocastic calculus, in Quantum Information, eds. T. Hida and K. Saito (World Scientific, 2005).

9. L. Accardi and R. Roschin, Renormalized squares of boson fields, Inf. Dim. Anal. Quantum Probab. Rel. Topics 8 (2005) 307.

10. P. Blasiak, K. A. Penson and A. I. Solomon, The general boson normal ordering problem, Phys. Lett. A 309 (2003) 198-205.

11. P. Śniady, Quadratic bosonic and free white noises, Commun. Math. Phys. 211 (2000) 615-628. 